\title{
Interkulturelle Dramapädagogik von Benedikt Kessler
}

\section{Manfred Schewe}

In der fremdsprachendidaktischen Fachdiskussion wird die Dramapädagogik zunehmend häufiger zum Bezugspunkt. Inzwischen ist ihr enormes Potenzial für die Sprach-, Literatur- und Kulturvermittlung in Forschungsarbeiten und Praxisberichten vielfach beleuchtet worden (vgl. dazu die umfangreiche Bibliographie auf der SCENARIO-Homepage). Inwiefern durch die Nutzung des Potenzials der Dramapädagogik speziell das interkulturelle Lernen effektiv gefördert werden kann, wurde bereits öfter thematisiert (vgl. z.B. den Sammelband von Byram/Fleming (1998)). Allerdings liegt nunmehr mit Benedikt Kesslers in drei Teile gegliederter, knapp 150seitiger Veröffentlichung ein erster Systematisierungsversuch vor. Im Sinne des Buchtitels ist er mit dem Anspruch verbunden, das Feld einer „Interkulturellen Dramapädagogik“ zu markieren.

Im ersten Teil thematisiert Kessler, Lehrer in den Fächern Englisch und Politik/Wirtschaft für das Lehramt an Gymnasien, wie im Laufe der letzten zwanzig Jahre der Aspekt des interkulturellen Lernens in der Fremdsprachendidaktik immer mehr an Bedeutung gewonnen hat und der kommunikative Fremdsprachenunterricht um die Dimension der Interkulturellen Kommunikativen Kompetenz (IKK) erweitert wurde. Er reibt sich dabei allerdings an der Tatsache, dass die damit einhergehenden theoretischen Ansprüche bisher nicht in eine überzeugende Unterrichtspraxis umgesetzt wurden. Seine Bestandsaufnahme mündet in die Feststellung,

dass die Verwirklichung interkultureller Kompetenzen im Allgemeinen und die Realisierung des umfassenden Ansatzes der IKK im Besonderen in weiter Ferne zu sein scheint. Allenthalben betont man die Bedeutung interkultureller Kompetenzen und die Pflicht des Fremdsprachenunterrichtes, diese auszubilden und zu fördern. Andererseits mangelt es an konkreten Hilfen für die Fremdsprachenlehrer, die mit der gewaltigen Aufgabe, interkulturelle kommunikative Kompetenz zu vermitteln, ohnehin oft schlichtweg überfordert sind. Folglich erscheint es wenig überraschend, dass die heutige Unterrichtspraxis den interkulturellen Postulaten der Lehrpläne kaum Rechnung tragen kann. (36)

Zur Lösung dieser Implementationsproblematik kann laut Kessler die Dramapädagogik signifikante Beiträge leisten, da sie die methodischen Mittel bereit hält, mit denen das interkulturelle Potenzial von literarischen Texten und 
Lehrwerkmaterialien ausgeschöpft werden kann, um Schüler auf interkulturelle Begegnungen gezielt vorzubereiten.

Im Teil II setzt sich Kessler mit Begriff und Geschichte der britischen Dramapädagogik auseinander und deckt dabei die wesentlichen Entwicklungsetappen ab. Er bemüht sich um eine differenzierte Darstellung verschiedener dramapädagogischer Konzepte, doch leserfreundlicher wäre es gewesen, wenn er Teile seiner kritischen Analyse nicht so häufig in langen Fußnoten untergebracht hätte. Ebenso hilfreich wäre auch eine deutlichere Unterscheidung zwischen Konzepten gewesen, die primär auf das Schulfach Drama ausgerichtet sind, und solchen, die sich auf die spezifische Situation des Fremd- und Zweitsprachenunterrichts beziehen, wobei z.B. das unerwähnte Process Drama-Konzept von Kao/O’Neill (1998) eine Brücke zwischen diesen beiden Bereichen darstellen könnte.

Auf alle Fälle gelingt es Kessler hervorragend, durch die sorgfältige Analyse theoretischer Schriften auf die gemeinsamen Anliegen (bzw. die „shared mission“, 46ff) von interkulturellem Lernen und Dramapädagogik aufmerksam $\mathrm{zu}$ machen, um in einem Folgekapitel (54 ff) heraus zu arbeiten, welche der „Dimensionen interkultureller Kompetenz" (affektiv-bewusstseinsorientierte, kognitive, prozedurale, linguistische) durch dramapädagogische Unterrichtsgestaltung besonders effektiv erreicht werden können, wobei er die affektivbewusstseinsorientierte Dimension um „das körperliche Bewusstsein“erweitert:

Der affektiv-bewusstseinsorientierten Dimension der IKK kann durch dramatisches Arbeiten daher eine ,Zusatzkompetenz', ein weiteres savoir, hinzugefügt werden, das ich als savoir percevoir bezeichnen möchte. Hierunter verstehe ich also einerseits die Bereitschaft dazu, Aufgeschlossenheit, Akzeptanz und Toleranz nicht nur mit Hilfe sprachlich-intellektueller, sondern auch physischer Zeichen zu äußern. Andererseits fasse ich es jedoch auch als physisch-kulturelles Bewusstsein auf, als die Bereitschaft, sich überhaupt mit der eigenen Körperlichkeit und der des anderen auseinanderzusetzen. Ein solches savoir percevoir würde demnach auch das savoir s'engager, die ,critical cultural awareness', insofern komplementieren, als es ein Bewusstsein für Sprache und Kultur ermöglichen würde, welches das Eigene und das Fremde nicht zu mentalen Erscheinungen reduziert, sondern diese als holistische, als geistige und körperliche Phänomene begreift. (57)

Er führt damit eine Fachdiskussion fort, zu der insbesondere Bräuer (2002) durch die Herausgabe des Sammelbandes Body and Language: Intercultural Learning through Drama beigetragen hat.

Zentral in Kesslers Konzept einer „Interkulturellen Dramapädagogik“ ist der gezielte Einsatz ausgewählter methodischer Mittel bzw. „dramatischer Konventionen“, die im Anhang aufgelistet werden und auf die jeweiligen „Dimensionen interkultureller Kompetenz" bezogen werden. Zur allgemeinen Zielorientierung des mit Hilfe solcher Konventionen gestalteten Fremdsprachenunterrichts wird die Realisierung eines „dritten Ortes“ als Ort der Vermittlung zwischen Eigenem und Fremdem, an dem ,aus den beiden Polen ,fremd' und ,eigen' bzw. ,unbekannt' und ,bekannt' etwas Neues, etwas Gemeinsames entsteht“" (90). 
Wie sich Kessler dies vorstellt, demonstriert er im dritten Teil seines Buches anhand eines Dramaprojektes, das er im Englischunterricht an einer Frankfurter Schule durchführte. Mit einer Schülergruppe (11. Jahrgangsstufe), in der 13 verschiedene kulturelle Hintergründe repräsentiert waren, wurde dramapädagogisch mit der Kurzgeschichte A Pair of Jeans (Qaisra Shahraz, 2005) gearbeitet. Durch die Auswertung von Reflexionsgesprächen und eines Fragebogens kommt Kessler zu dem Ergebnis, dass seine Unterrichtsversuche die vorangegangenen theoretischen Ausführungen bestätigen, allerdings bestimmte Aspekte weiterer Erforschung bedürfen. So stellt er z.B. selbstkritisch fest, dass die Förderung eines Bewusstseins für die eigene Körperlichkeit (und die des anderen) gezielterer Herangehensweisen bedarf und überhaupt $\mathrm{zu}$ klären wäre, ob männliche Lerner zögerlicher auf die körperbezogene dramapädagogische Arbeit reagieren als weibliche. Konsequent und begrüßenswert ist, dass Kessler seine theoretischen Ausführungen praktisch zu untermauern sucht, allerdings hätte die Evaluation der Praxis stärker in ein begründetes Forschungskonzept, etwa ein Aktionsforschungskonzept, eingebunden werden können.

Benedikt Kessler legt mit dieser Arbeit eine wichtige Grundlage für künftige Fachdiskussionen, indem er die Schnittmenge zwischen interkulturellem Lernen und Dramapädagogik deutlich in den Blick rückt und anhand der Beschreibung und Auswertung seiner eigenen Praxis Lehrern und Lehrerinnen Mut macht zur Entwicklung von Praxismodellen. Nur auf diese Weise wird sich schließlich auch der theoretische Anspruch einer „Interkulturellen Dramapädagogik" überprüfen lassen. Dass dieses Konzept auch theoretisch noch erweitert werden kann, belegt Katja Frimbergers Beitrag Towards a Pedagogy of Strangeness: Exploring the Potential of Strangeness for Foreign Language Education, der in dieser Ausgabe abgedruckt ist und hier herunter geladen werden kann. Darin thematisiert sie, wie theatertheoretische und -praktische Konzepte, insbesondere auch Brechtsche Arbeitsweisen, gewinnbringend auf die Praxis des fremdsprachlichen Unterrichts übertragen werden können, um sinnvoll auf den Umgang mit Fremdkultur vorzubereiten und entsprechende Handlungskompetenzen auszubilden.

Wer sich für die weitere Ausmessung des Feldes einer „Interkulturellen Dramapädagogik“ interessiert, sei in Verbindung mit dieser Besprechung Benedikt Kessler und Almut Küppers' Artikel Dramapädagogik, interkulturelle Kompetenz und holistisches Fremdsprachenlernen empfohlen. Er erschien in Ausgabe 2/2008 dieser Zeitschrift und kann hier direkt herunter geladen werden.

\section{Bibliographie}

Bräuer, Gerd (ed.) (2002): Body and Language. Intercultural Learning through Drama. Westport, Connecticut / London, Ablex Publishing Corporation 
Byram, Michael \& Fleming, Michael (eds.) (1998): Language Learning in Intercultural Perspective. Approaches through Drama and Ethnography. Cambridge, Cambridge University Press

Kao, Shin-Mei \& O'Neill, Cecily (1998): Words into Worlds. Learning a Second Language through Process Drama. Stamford, CT / London: Ablex Publishing Kessler, Benedikt (2008): Interkulturelle Dramapädagogik. Dramatische Arbeit als Vehikel des interkulturellen Lernens im Fremdsprachenunterricht. Frankfurt/Main: Peter Lang 